\title{
Available evidence does not support use of oxalates for dentine hypersensitivity
}

\author{
Abstracted from \\ Cunha-Cruz J, Stout JR, Heaton LJ, Wataha JC; Northwest PRECEDENT \\ Dentin hypersensitivity and oxalates: a Systematic Review. \\ J Dent Res 2011; 90: 304-310. Epub 2010 Dec 29. \\ Address for correspondence \\ Cunha-Cruz J, Dental Public Health Sciences, Box 357475, School of Dentistry, \\ University of Washington, Seattle, WA 98195-7475, USA. E-mail: silvajcc@u.washington.edu
}

\section{Question: Do oxalates desensitise hypersensitive dentine?}

Data sources Medline, CENTRAL, LILACS, TRIP Database, National Guideline Clearinghouse, OPENSigle and a number of other medical / dental databases and websites, as well as reference lists.

Study selection Randomised controlled trials and controlled clinical trials in any language that compared oxalates with placebo or no treatment were eligible for inclusion.

Data extraction and synthesis Three reviewers extracted data with two people assessing quality independently using the Cochrane risk of bias domains. Standardized mean differences (SMD) were estimated by random-effects meta-analysis and heterogeneity between studies was quantified with the $\mathrm{I}^{2}$-statistic.

Results 12 reports were included. Interventions were diverse and included: monohydrogen-monopotassium oxalate, ferric oxalate, di-potassium oxalate, or oxalate containing pre-polymerized resin, and combinations of monohydrogen-monopotassium and di-potassium oxalate. Follow up intervals varied between immediate to 1 year. Hypersensitivity was elicited by tactile, evaporative or thermal stimuli, and a number of outcomes used to measure pain. Risk of bias was high in a number of studies and statistical heterogeneity high. Based on 187 and 179 units (patients or teeth) in the intervention and placebo groups from seven studies, the summary SMD for 3\% monohydrogenmonopotassium oxalate was -0.71 (95\% Confidence Interval (Cl):$1.48,0.06)$, which suggests it may not be beneficial. There was no improvement with the other interventions.

Conclusions The review identified no evidence to indicate benefit from treating dentine hypersensitivity with oxalates beyond a placebo effect.

\section{Commentary}

Dentine hypersensitivity (DH) is a problem which has challenged both researchers and clinicians and bothered patients for many years, and for which no effective treatment has yet been identified. This review of 12 clinical trials on the efficacy of oxalates on $\mathrm{DH}$, was conducted using the Cochrane methodology including only randomised controlled trials or controlled trials. The findings are summarised using a meta-analysis.

Clinical trials of agents against DH present several methodological problems, some of which are common to all clinical trials, and some of which are specific to clinical trials on anti-DH agents. Amongst the former, the authors found considerable variation in risk-of-bias, as assessed using standardised methods. Amongst the latter is the measurement of pain, which has been measured in a number of different ways, eg after tactile or thermal stimulations, using a standardised air blast, or as reported in connection with daily 'routine activities', (eating, drinking and tooth cleaning), by the patients. Some trials use only one of these methods, while other trials use different combinations of methods. This variation in outcome measure causes difficulties when it comes to comparing the trials and synthesising the results.

The experimental design of the trails also differed: nine of the 12 trials used the split-mouth design and the remaining three used a parallel-group design. The methodological problems associated with the split-mouth design have been reviewed recently, ${ }^{1}$ and the possibility of a carry-over effect from one side of the mouth to the other has been pointed out as an important risk-of-bias. To what extent this factor has been taken into account in the trials is not reported.

The meta-analysis showed that only one of the oxalate compounds (3\% monohydrogen monopotassium oxalate) approached a statistically significant effect on DH. However, this conclusion is based on seven trials, of which three showed no effect, or an effect in favour of placebo, three showed marginal effect in favour of oxalate, while one trial showed a marked effect in favour of oxalate. This trial however, did not report the age of the subjects, included a small number of observations (15), and presented only patient-reported effect on routine activities.

At the end of the manuscript the authors conclude that 'available evidence suggests that oxalates are not effective in decreasing DH ...'. When assessing evidence, it is however often repeated that 'no evidence of effect' is not the same as 'evidence of no effect'. With this in mind, the last sentence in the abstract is a more appropriate summary of the findings of this review: '... available evidence currently does not support the recommendation of dentine hypersensitivity treatment with oxalates'.

\section{Practice points}

- No strong evidence is available to support the use of oxalates for treatment of dentine hypersensitivity.

\section{Sven Poulsen}

Department of Pediatric Dentistry, School of Dentistry, Faculty of Health Sciences, University of Aarhus, Aarhus, Denmark.

1 Lesaffre $\mathrm{E}$, Philstrom B, Needleman I, Worthington $\mathrm{H}$. The design and analysis of split-mouth studies: what statisticians and clinicians should know. Stat Med 2009; 28: $3470-3482$.

Evidence-Based Dentistry (2011) 12, 47. doi:10.1038/sj.ebd.6400792 